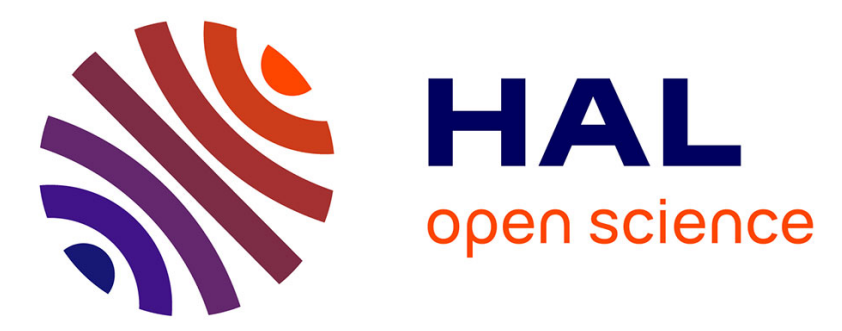

\title{
Passive earth pressures in the presence of hydraulic gradients
}

\author{
Abdul-Hamid Soubra, Richard Kastner, A. Benmansour
}

\section{To cite this version:}

Abdul-Hamid Soubra, Richard Kastner, A. Benmansour. Passive earth pressures in the presence of hydraulic gradients. Géotechnique, The Institution of Civil Engineers, 1999, 49 (3), pp.319-330. 10.1680/geot.1999.49.3.319 . hal-01007178

\section{HAL Id: hal-01007178 https://hal.science/hal-01007178}

Submitted on 28 Jan 2017

HAL is a multi-disciplinary open access archive for the deposit and dissemination of scientific research documents, whether they are published or not. The documents may come from teaching and research institutions in France or abroad, or from public or private research centers.
L'archive ouverte pluridisciplinaire HAL, est destinée au dépôt et à la diffusion de documents scientifiques de niveau recherche, publiés ou non, émanant des établissements d'enseignement et de recherche français ou étrangers, des laboratoires publics ou privés. 


\title{
Passive earth pressures in the presence of hydraulic gradients
}

\author{
A. H. SOUBRA, ${ }^{*}$ R. KASTNER $\dagger$ and A. BENMANSOUR* \\ * Ecole Nationale Supérieure des Arts et Industries de Strasbourg \\ $\dagger$ Institut National des Sciences Appliquées de Lyon.
}

The paper describes a variational approach applied to the limit equilibrium method for calculating the effective passive pressures of a cohesionless soil, taking into consideration the seepage flow. It is shown that in the general case of non-homogeneous and non-isotropic hydraulic properties of the soil medium, the shape of the slip surface which verifies the three limiting equilibrium equations of the soil mass at failure is a log-spiral. It is also shown that the passive earth pressure calculation is independent of the normal stress distribution along this surface. The variational limit equilibrium method is equivalent to the upper bound method in limit analysis for a rotational log-spiral mechanism. Numerical results of the coefficients of passive earth pressures in the presence of seepage flow are presented and discussed.

KEYWORDS: earth pressure; filters; limit state design/analysis; pore pressures; seepage; sheet piles and cofferdams.

\section{INTRODUCTION}

The design of deep sheeted excavations is often dominated by the flow of water around the sheet piles. The seepage flow influences the stability of the excavation where bulk heave or piping may occur. While the piping takes place at the excava tion level, the heaving is more catastrophic and its risk is usually evaluated by considering a rectangu lar failure mechanism adjacent to the wall (Terzaghi, 1943). The vertical force equilibrium of this soil mass is then considered by neglecting the vertical frictional forces along the vertical faces of this mechanism.

Based on laboratory model tests, Kastner (1982) has shown that the failure of the sheet piling structures in the presence of seepage flow is not only due to the heaving phenomenon but may also
Cet article présente une approche variationnelle appliquée à la méthode du prisme de rupture permettant le calcul de la pression passive effective des terres en présence d'écoulement dans le cas d'un sol purement pulvérulent. On montre que dans le cas général d'un sol aux propriétés hydrauliques non homogènes et non isotropes, la forme de la surface de rupture qui vérifie les trois équations d'équilibre est une spirale logarithmique. Nous montrons aussi que le calcul de la pression passive ne dépend pas de la distribution des contraintes normales agissant le long de cette surface. La méthode du prisme de rupture variationnelle est équivalente à la méthode de la borne supérieure en analyse limite pour un mécanisme rotationnel en spirale logarithmique. Des valeurs numériques du coefficient de butée en présence d'écoulement sont présentées et discutées.

occur due to the reduction of the passive earth pressures in front of the wall. Our aim in this paper is to propose an outline for the calculation of the effective passive pressures, taking into ac count the seepage forces.

Looking for a simple model capable of correctly describing the behaviour of soil in the passive state in the presence of seepage flow, we opted for the limit equilibrium method. This approach is based on a priori hypotheses concerning the shape of the slip surface (kinematic function) and the normal stress distribution (static function) along this sur face. The variational approach applied to the limit equilibrium method has been employed in order to avoid the restrictions of such a priori hypotheses.

OVERVIEW OF PREVIOUS VARIATIONAL ANALYSIS

The variational limit equilibrium method has been used by several authors in geotechnical en gineering. Kopacsy (1957) applied this approach to the three dimensional slope stability problem; however, no explicit solution is offered. Several investigators, for example Dorfman (1965), Garber (1973), Revilla \& Castillo (1977) and Ly (1979), have used the calculus of variations to 
avoid introducing an assumption concerning the shape of the slip surface but they made an assump tion concerning the normal stress distribution along this surface. A more interesting analysis consists of finding the two unknown functions without any $a$ priori assumptions. Thus, Baker \& Garber (1977, 1978) applied the variational approach to the two dimensional slope stability problem, and Garber \& Baker (1977) and Castillo \& Luceno (1978) ap plied this approach to the problem of the bearing capacity of a strip footing. Then, Garber \& Baker (1979) treated the problems of slope stability, bear ing capacity and earth pressure distribution in a unified manner as a single problem. Later, this method was used by Leshchinsky et al. (1985), Ugai (1985) and Leshchinsky \& Baker (1986) to study the three dimensional slope stability problem, and by Leshchinsky \& Reinschmidt (1985), who applied it to the reinforced slope stability problem. Finally, Leshchinsky \& San (1994) applied the variational limit equilibrium method to the seismic stability of slopes, and presented interesting results in the form of design charts. It is to be noted here that the variational approach has been the subject of some controversy. Particularly, the work by Castillo \& Luceno (1982) shows that the functional has no minimum. Notice, however, that experience indicates that the slip surface determined by the variational analysis reasonably duplicates reality (Leshchinsky \& San, 1994). Consequently, the solutions given by the variational limit equilibrium method are very interesting. We present in the following the application of this method to the passive earth pressure problem, taking into account the seepage forces.

\section{VARIATIONAL APPROACH OF THE PASSIVE EARTH PRESSURE PROBLEM}

Figure 1 shows a double walled cofferdam sub jected to a seepage flow where $H$ is the total head loss and $u(x, y)$ represents the distribution of the pore water pressures in the soil medium.

The assumptions made in the analysis can be summarized as follows:

(a) The soil is cohesionless. It is homogeneous and isotropic with respect to its angle of internal friction $\phi$.

(b) The soil medium is non homogeneous and non isotropic with respect to the hydraulic properties. It is composed of $n$ permeable layers overlying impermeable rock. Each layer is characterized by its coefficients of per meability $K_{\mathrm{h} i}$ and $K_{\mathrm{v} i}$.

(c) The breadth $B_{0}$ (Fig. 1) is large enough so that there is no interaction of the two failure mechanisms which develop in front of the two walls of the cofferdam.

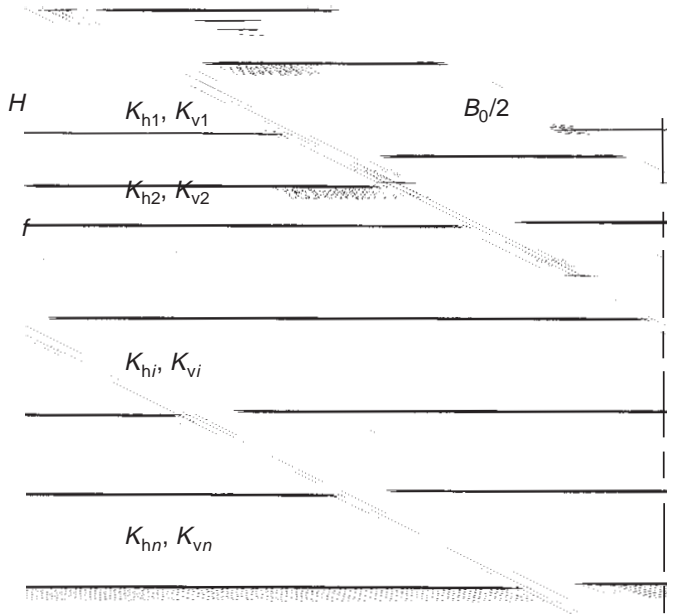

Fig. 1. Double-walled cofferdam in a multi-layered soil medium

(d) The resultant $P_{\mathrm{P}}$ of the effective passive pressures is assumed to act at the bottom third of the penetration depth (Fig. 2(a)). This force can be expressed as follows:

$$
P_{\mathrm{P}}=K_{\mathrm{P}} \frac{\gamma^{\prime} f^{2}}{2}
$$

where $K_{\mathrm{p}}$ is the coefficient of passive earth pres sure in the presence of seepage flow, $\gamma^{\prime}$ is the submerged unit weight of the soil, and $f$ is the penetration depth.

The variational approach is briefly presented in this paper. For more details, refer to Soubra (1989). Fig. 2(a) illustrates the formulated problem and shows the notation used. A potential slip sur face $y(x)$ is subjected to a total normal stress $\sigma(x)$. Both functions $y(x)$ and $\sigma(x)$ are assumed to be continuous. Using Coulomb's failure criterion $\tau(x)=\left[\begin{array}{ll}\sigma(x) & u(x)\end{array}\right] \tan \phi=\sigma^{\prime}(x) \tan \phi$, the global limiting equilibrium equations for the soil mass (Fig. 2(b)) can be written as

$$
\begin{aligned}
& P_{\mathrm{p}} \cos \delta+U_{1}=\int_{x_{0}}^{x_{1}}\left[\sigma^{\prime}(\tan \phi+\dot{y})+u \dot{y}\right] \mathrm{d} x \\
& P_{\mathrm{p}} \sin \delta= \\
& \quad \int_{x_{0}}^{x_{1}}\left[\begin{array}{llll}
\sigma^{\prime}(1 & \tan \phi \cdot \dot{y})+u & \left.\gamma_{\mathrm{sat}}(f \quad y)\right] \mathrm{d} x & (2 \mathrm{~b}) \\
P_{\mathrm{p}} \cos \delta X_{1}+U_{1} X_{2}=\int_{x_{0}}^{x_{1}}\left[\begin{array}{lll}
\sigma^{\prime}(1 & \tan \phi \cdot \dot{y}) x
\end{array}\right. \\
\quad+\sigma^{\prime}(\tan \phi+\dot{y}) y+u x+u y \dot{y} & \left.\gamma_{\mathrm{sat}}(f \quad r) x\right] \mathrm{d} x
\end{array}\right.
\end{aligned}
$$

Notice that one of the endpoints $x_{0}$ is null 


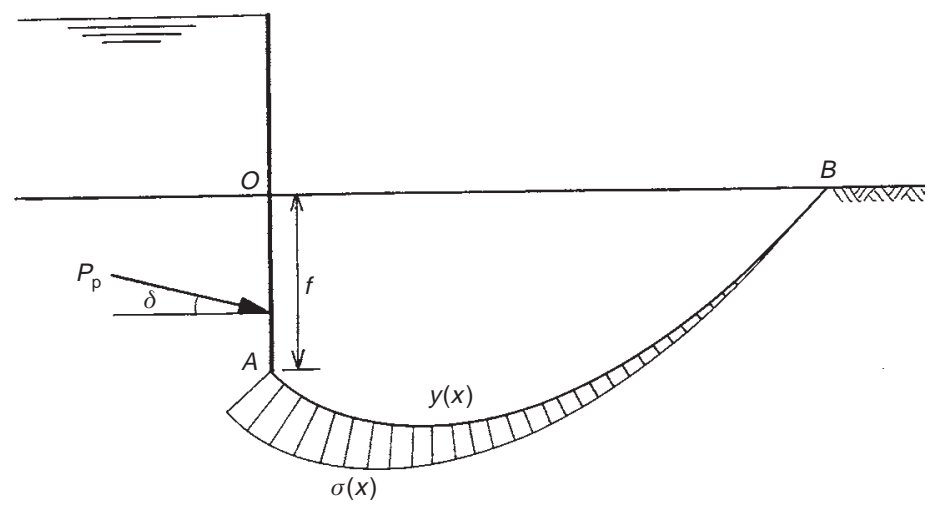

(a)

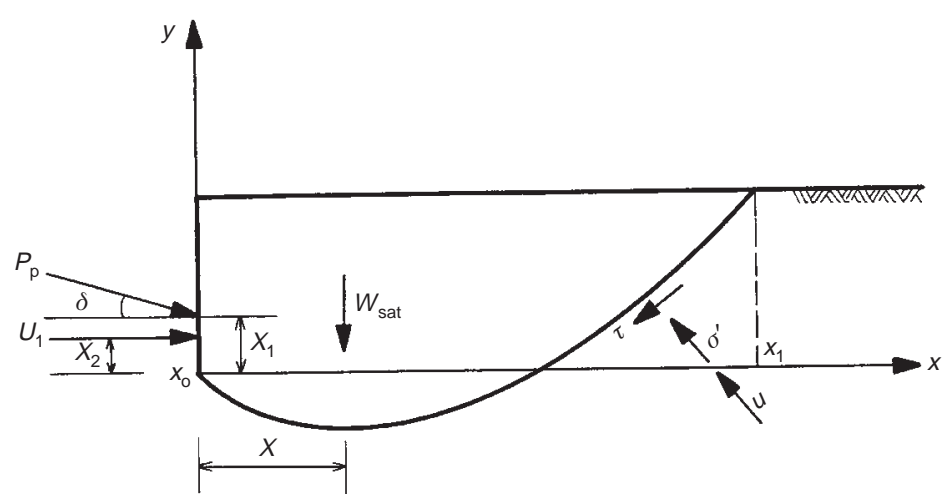

(b)

Fig. 2. (a) Slip surface for passive earth pressure analysis. (b) Free body diagram

(Fig. 2(b)), and the other (i.e. $x_{1}$ ), is variable. Observing equations (2a), (2b) and (2c), one rea lizes that the effective passive force $P_{\mathrm{p}}$ is a func tional of two functions, $y(x)$ and $\sigma^{\prime}(x)$. The mathematical problem of the passive earth pressure is to find those functions which give the minimum value of the passive force functional and simulta neously satisfy all three equations of limiting equi librium (equations (2a), (2b) and (2c)).

Using equation (2b) to define $P_{\mathrm{p}}$, while consider ing the other two equilibrium equations (equations (2a) and (2c)) as constraints (i.e. equations that must be satisfied), the passive earth pressure problem is a variational isoparametric one with a variable end point. This variational problem is equivalent to the minimization of an auxiliary functional $G$ :

$$
G=L_{0}+\lambda_{1} L_{1}+\lambda_{2} L_{2}
$$

where $L_{0}, L_{1}$ and $L_{2}$ are given as follows:

$$
\begin{aligned}
& L_{0}=\sigma^{\prime}(1 \quad \tan \phi \dot{y})+u \quad \gamma_{\text {sat }}(f \quad y) \\
& L_{1}=\sigma^{\prime}(\tan \phi+\dot{y})+u \dot{y} \\
& L_{2}=\sigma^{\prime}(1 \quad \tan \phi \dot{y}) x+\sigma^{\prime}(\tan \phi+\dot{y}) y \\
& +u x+u y \dot{y} \quad \gamma_{\text {sat }}(f \quad y) x
\end{aligned}
$$

$\lambda_{1}$ and $\lambda_{2}$ are the Lagrange undetermined multi pliers. Finally, the two extremal functions $y(x)$ and $\sigma^{\prime}(x)$ must satisfy the following conditions:

(a) The system of Euler's differential equations for the functional $G$ :

$$
\begin{aligned}
& \frac{\partial G}{\partial \sigma^{\prime}}=\frac{\mathrm{d}}{\mathrm{d} x} \frac{\partial G}{\partial \dot{\sigma}^{\prime}} \\
& \frac{\partial G}{\partial y}=\frac{\mathrm{d}}{\mathrm{d} x} \frac{\partial G}{\partial \dot{y}}
\end{aligned}
$$

(b) The constraint equations.

(c) The boundary conditions: at the fixed endpoint 
$A$, we have $x_{A}=y_{A}=0$. At the variable end point $B$, a variational condition must be satis fied. This condition is called the 'transversality condition' and it can be written as follows:

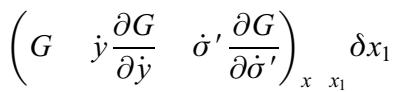

$$
\begin{aligned}
& +\left(\frac{\partial G}{\partial \dot{\sigma}^{\prime}}\right)_{x x_{1}} \delta \sigma_{1}^{\prime}+\left(\frac{\partial G}{\partial \dot{y}}\right)_{x x_{1}} \delta y_{1}=0
\end{aligned}
$$

where $\delta$ is a variational operator.

\section{The first Euler equation}

Combining equations (3) and (5a) yields a dif ferential equation. The solution of this equation in a polar coordinate system is a log spiral (Fig. 3) whose equation is given as follows:

$$
r=r_{0} \exp \left(\theta \quad \theta_{0}\right) \tan \phi
$$

Note that the log spiral function has a particular property, that the resultant of the forces $\left(\sigma^{\prime} \mathrm{d} l\right)$ and $\left(\tan \phi \sigma^{\prime} \mathrm{d} l\right)$ passes through the pole of the spiral. Hence, the moment equation about the pole is independent of the stress distribution $\sigma^{\prime}(x)$, and may be used for the determination of the effective passive force. The two remaining equilibrium equa tions may be satisfied by every $\sigma^{\prime}(x)$ distribution that has two degrees of freedom. Thus, one has to find the critical $\theta_{0}$ and $\theta_{1}$ angles which satisfy the moment equilibrium equation and give the mini mum value of the effective passive force $P_{\mathrm{p}}$. This is done by a two dimensional minimization proce dure of $P_{\mathrm{p}}$ with respect to $\theta_{0}$ and $\theta_{1}$.

The independence of the effective passive force from the normal stress distribution can also be shown, due to the special property of the present functional. This functional can be written as fol lows:

$$
G=\sigma^{\prime} f(x, y, \dot{y})+g(x, y, \dot{y})
$$

$G$ is linear in $\sigma^{\prime}$ and is independent of $\dot{\sigma}^{\prime}$. The first Euler equation implies that $f(x, y, \dot{y})=0$. Substituting this equation into equation (8), one can see that this functional becomes independent of $\sigma^{\prime}$ as follows: $G=g(x, y, \dot{y})$. This result is a direct consequence of the shape of the slip surface. Thus, the first Euler equation transforms the pas sive functional from a functional of two unknown functions to a functional of a single function. Therefore, it is possible to solve the passive earth pressure problem by simply minimizing the new functional $G$ without specifying the normal stress distribution. Finally, it is easy to see that the moment equation of the rotational log spiral me chanism around the centre is identical to the work equation for the same mechanism in the upper bound method in limit analysis. Thus, solving the passive earth pressure problem by writing the moment equation around the centre of the log spiral will give an upper bound solution of the exact solution for an associated flow rule Coulomb material.

\section{The second Euler equation and the transversality condition}

Since the aim of this study is the determination of the critical effective passive force, the results obtained so far are enough to solve the problem. Indeed, it has been shown by Baker \& Garber (1977) that the second Euler equation and the transversality condition give the normal stress dis tribution. In this work, this is also the case, but since the $\sigma^{\prime}(x)$ distribution is not necessary to assess the effective passive force, we will not express these equations.

\section{Existence of a minimum of the functional}

The second variation of the passive earth func tional shows that this functional is degenerated.

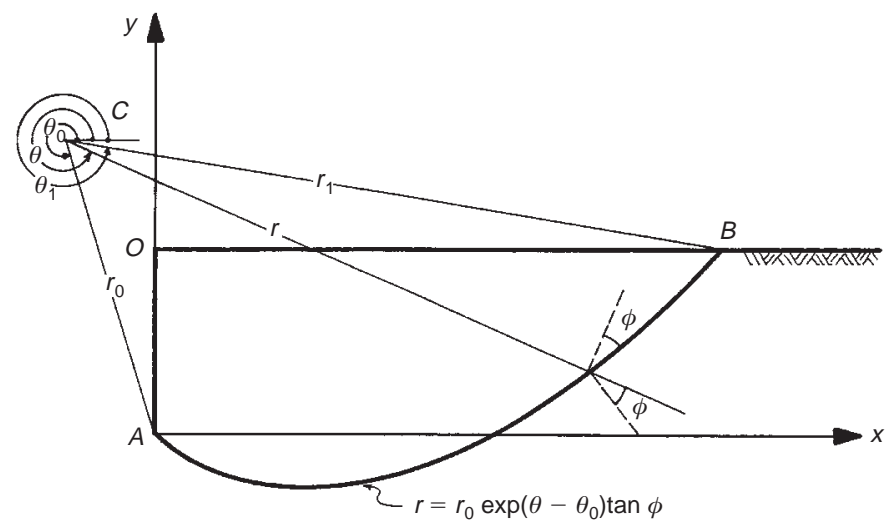

Fig. 3. Log-spiral slip surface for passive earth pressure analysis 
Thus, we cannot say mathematically that there is a minimum (Castillo \& Luceno, 1982). This diffi culty concerning the existence of a minimum can be overcome due to the equivalence between the variational limit equilibrium method and the upper bound method in limit analysis for a rotational mechanism as has been shown before. This equiva lence has been established in a more general way by Castillo \& Luceno (1983) and Leshchinsky et al. (1985).

\section{CALCULATION SCHEME OF THE PASSIVE EARTH PRESSURES}

As mentioned before, the solution of the passive earth pressure problem by a variational limit equi librium method is equivalent to saying that the equation of moment equilibrium must be satisfied for the soil mass bounded by the $\log$ spiral and the ground surface. As shown in Fig. (2b), the forces acting on the collapse mechanism are $(a)$ the saturated weight of the soil mass between the $\log$ spiral surface and the ground surface, $(b)$ the effective passive force $P_{\mathrm{p}}$ which is inclined at $\delta$ to the normal of the sheet pile, $(c)$ the pore water pressures along the penetration depth and the log spiral surface, and $(d)$ the effective normal and tangential stress distributions along $y(x)$. The moment equilibrium equation can be written as follows:

$$
\begin{aligned}
& W_{\text {sat }}\left(r_{0} \cos \theta_{0}+X\right)+P_{\mathrm{p}} \sin \delta\left(r_{0} \cos \theta_{0}\right) \\
& \quad=P_{\mathrm{p}} \cos \delta\left(\begin{array}{cc}
r_{0} \sin \theta_{0} & \frac{f}{3}
\end{array}\right)+M_{1}+M_{2}
\end{aligned}
$$

where $M_{1}$ and $M_{2}$ represent the moments of the force $U_{1}$ and of the pore water pressures along $y(x)$ respectively. They are given as follows:

$$
\begin{aligned}
M_{1}=\int_{0}^{f} u( & \left.r_{0} \sin \theta_{0} \quad y\right) \mathrm{d} y \\
& =r_{0} \sin \theta_{0} \int_{0}^{f} u \mathrm{~d} y \quad \int_{0}^{f} u y \mathrm{~d} y
\end{aligned}
$$

$$
\begin{aligned}
M_{2}=\int_{\theta_{0}}^{\theta_{1}}(u \sin \phi) & \frac{r \mathrm{~d} \theta}{\cos \phi} r \\
= & r_{0}^{2} \tan \phi \exp \left(2 \theta_{0} \tan \phi\right) \\
& \times \int_{\theta_{0}}^{\theta_{1}} u \exp (2 \theta \tan \phi) \mathrm{d} \theta
\end{aligned}
$$

From equation (9), one can easily see that $P_{\mathrm{p}}$ is a function of the two parameters $\theta_{0}$ and $\theta_{1}$ which completely describe the failure mechanism. The most critical $K_{\mathrm{p}}$ value is obtained by a minimiza tion procedure of the $K_{\mathrm{p}}$ coefficient given by equa tion (1), that is $\left[K_{\mathrm{P}}=\left(2 P_{\mathrm{P}}\right) /\left(\gamma^{\prime} f^{2}\right)\right]$ with respect to the two parameters mentioned above. A compu ter program has been developed for assessing the minimal $K_{\mathrm{p}}$ values and the corresponding critical slip surfaces.

\section{NUMERICAL RESULTS OF THE PASSIVE EARTH PRESSURES \\ Case of no seepage flow}

Table 1 compares the passive earth pressure coefficient $K_{\mathrm{p}}$ for $\phi=40^{\circ}$ and $\delta / \phi=1 / 2 \mathrm{ob}$ tained from the present analysis with that of other authors in the case of no seepage flow. The com parison of the present upper bound solution with the upper and lower bound solutions given respec tively by Chen \& Rosenfarb (1973) and Lysmer (1970) shows that the difference with the lower bound solution is smaller than $3 \%$, which means that the present solution is very close to the exact solution for an associated flow rule Coulomb material. On the other hand, the currently accepted values given by Sokolovski (1960) and Caquot \& Kérisel (1948) lie in the range between the best upper and lower bound solutions given by the limit analysis method. Finally, the comparison between the present result and the ones given by the limit equilibrium methods (Coulomb, 1776; Shields \& Tolunay, 1973) shows that the traditional limit equilibrium method may greatly overestimate or underestimate the passive earth pressure coeffi cients due to the a priori assumptions concerning

Table 1. Passive earth pressure coefficient $K_{\mathrm{p}}$ as given by different authors for $\phi \quad 40^{\circ}$, $\boldsymbol{\delta} / \boldsymbol{\phi} \quad \mathbf{1} / \mathbf{2}$

\begin{tabular}{l|l|r}
\hline & \multicolumn{1}{|c}{ Authors } & \multicolumn{1}{c}{$K_{\mathrm{p}}$} \\
\hline Limit equilibrium methods & Coulomb (1776) & $11 \cdot 77$ \\
Slip line methods & Shields \& Tolunay (1973) & $8 \cdot 30$ \\
& Caquot \& Kérisel (1948) & $9 \cdot 60$ \\
Upper bound methods in limit analysis & Sokolovski (1960) & $9 \cdot 68$ \\
& Chen \& Rosenfarb (1973) & $10 \cdot 10$ \\
Lower bound methods in limit analysis & Present solution & $9 \cdot 81$ \\
& Lysmer (1970) & $9 \cdot 54$ \\
\hline
\end{tabular}


the shape of the slip surface and the normal stress distribution along this surface.

\section{Case of seepage flow}

From equation (9), the determination of the effective passive force $P_{\mathrm{P}}$ requires the determina tion of the terms $M_{1}$ and $M_{2}$. The hydraulic head distribution $\varphi(x, y, z)$ in the soil medium is gov erned by the following equation:

$$
\frac{\partial}{\partial x}\left(K_{x} \frac{\partial \varphi}{\partial x}\right)+\frac{\partial}{\partial y}\left(K_{y} \frac{\partial \varphi}{\partial y}\right)+\frac{\partial}{\partial z}\left(K_{z} \frac{\partial \varphi}{\partial z}\right)=0
$$

In some simple cases, such as the case of the single sheet pile driven into a semi infinite homo geneous soil medium, the pore water pressure distribution is given analytically (Soubra \& Kast ner, 1992). For more complex geometry or for a multi layered soil medium with different coeffi cients of permeability, the pore water pressure dis tribution cannot be known analytically and it requires a numerical resolution of equation (12).

For the double walled cofferdam considered in this paper, the numerical method used for the determination of the potential field in the soil medium is the well known finite difference meth od where the differential equation (equation 12) is approximated by a finite difference equation. The boundary conditions used are shown in Fig. 4. The medium is discretized by a rectangular mesh (Fig. 5). The finite difference equations written at the different nodes form a system of linear equations whose unknowns are the values of the hydraulic head at the nodes. This system is solved by the Gauss Seidel method, using over relaxation in order to accelerate the rate of convergence.

The determination of the moments $M_{1}$ and $M_{2}$ has been made by numerical integration using the Gaussian quadrature method where the pore water pressures along both the sheet pile and the log spiral surface are determined by numerical interpolation. Let us pass now to the presentation of some numerical results obtained from the com

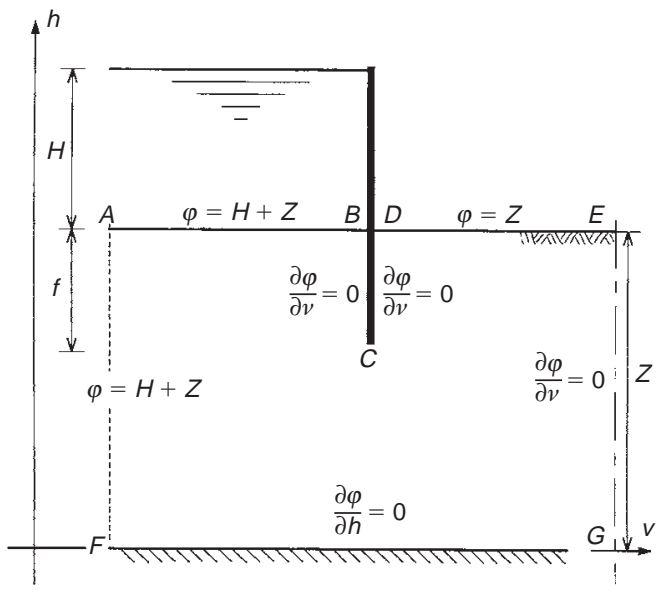

Fig. 4. Boundary conditions for the seepage flow of the double-walled cofferdam

puter program. Notice that all subsequent results concern the case when $\gamma_{\text {sat }} / \gamma_{\mathrm{w}}=2$ and $B_{0} / 2=$ $10 \mathrm{~m}$ (Fig. 1).

Case of a homogeneous and isotropic soil medium

Case of a single layer of infinite depth. Soubra \& Kastner (1992) published the results of the passive earth pressure coefficients in the presence of seepage flow in the case of a single sheet pile wall driven into a homogeneous and isotropic semi infinite soil medium where the hydraulic head can be known analytically. Note that the same results have also been obtained by the present analysis using the finite difference method for the determi nation of the hydraulic heads.

Figure 6 shows the variation of the passive earth pressure coefficient with $H / f$ for $\phi=30^{\circ}$ and for four values of $\delta / \phi(\delta / \phi=0,1 / 3,1 / 2,2 / 3)$. For a zero $K_{\mathrm{p}}$ value, the corresponding $H / f$ value is the same for different $\delta / \phi$ values $(H / f=2.78)$. This means that the angle of friction at the soil structure interface has no effect on the $H / f$ value causing failure by heaving. This fact can

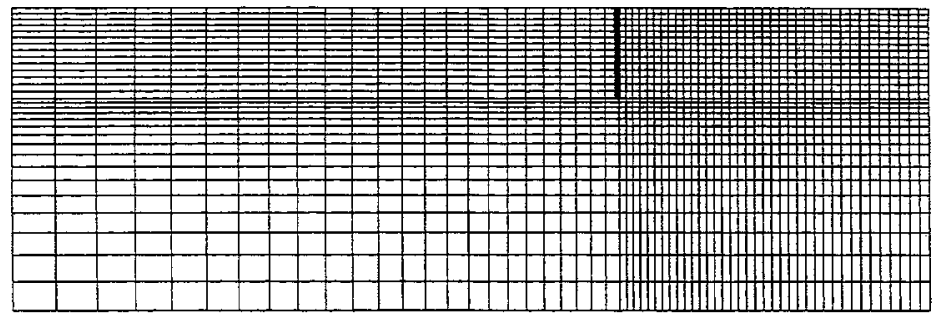

Fig. 5. Finite difference mesh 


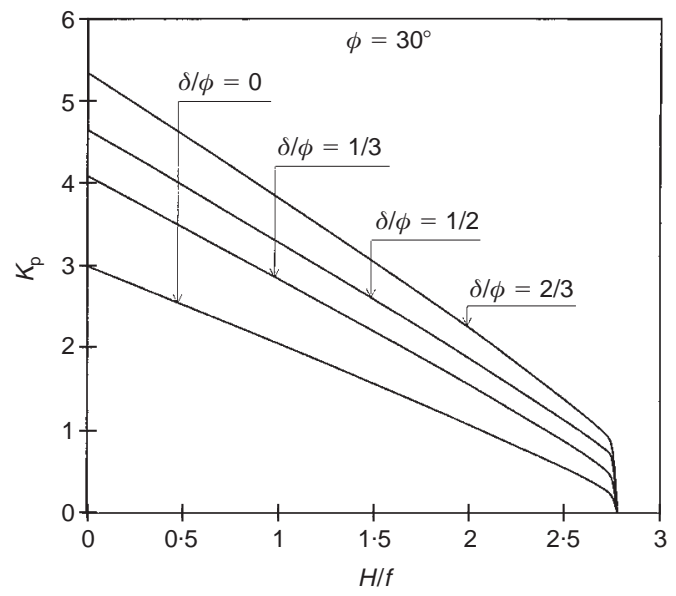

Fig. 6. $K_{\mathrm{p}}$ versus $H / f$ for $\phi \quad 30^{\circ}$ and $\delta / \phi \quad 0,1 / 3$, $1 / 2$ and $2 / 3$ in the case of a homogeneous isotropic semi-infinite medium

be explained as follows: when the effective passive force vanishes, there is no interaction at the soil structure interface and we have the tradi tional heaving phenomenon. For the same case, Terzaghi's approach gives a value of $H / f$ at fail ure equal to $2 \cdot 82$. Furthermore, the piping phe nomenon which appears for the critical hydraulic gradient at the point $D$ (Fig. 4) occurs for a value of $H / f$ equal to $3 \cdot 14$. It should be mentioned that the numerical results have shown that in the case of a homogeneous and isotropic semi infinite soil medium, the failure by heaving will occur before the piping phenomenon as long as $\phi$ is smaller than $45^{\circ}$.

Figure 7 shows some charts of the variation of the passive earth pressure coefficient as a function of $H / f$ for different values of $\phi\left(\phi=20^{\circ}, 25^{\circ}\right.$, $\left.30^{\circ}, 35^{\circ}, 40^{\circ}\right)$ and $\delta / \phi(\delta / \phi=0,1 / 3,2 / 3)$. From these figures, the reduction of this coefficient is quasi linear for the $H / f$ values varying from 0 to $2 \cdot 5$.

Finally, it should be mentioned that the $K_{\mathrm{p}}$ value increases with $\gamma_{\text {sat }}$. This increase is to be expected, since the soil weight has the favourable effect of increasing the stability of the soil mass in front of the sheet pile. For example, when $\phi=35^{\circ}, \delta / \phi$ $=2 / 3$ and $H / f=2$, the increase of the passive earth pressure coefficient attains $22 \%$ when $\gamma_{\text {sat }} / \gamma_{\mathrm{w}}$ increases from 2 to $2 \cdot 2$.

Fig. 7. $K_{\mathrm{p}}$ versus $H / f$ for different values of $\phi$ and $\delta$ in the case of a homogeneous isotropic semi-infinite medium

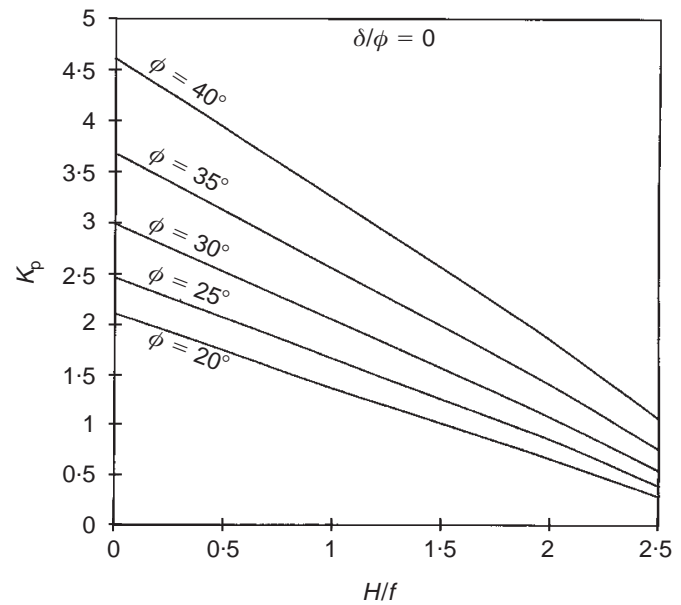

(a)

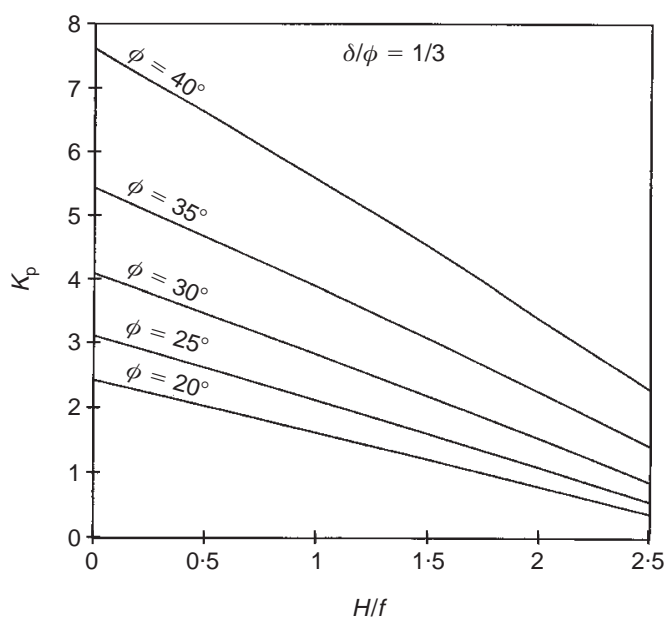

(b)

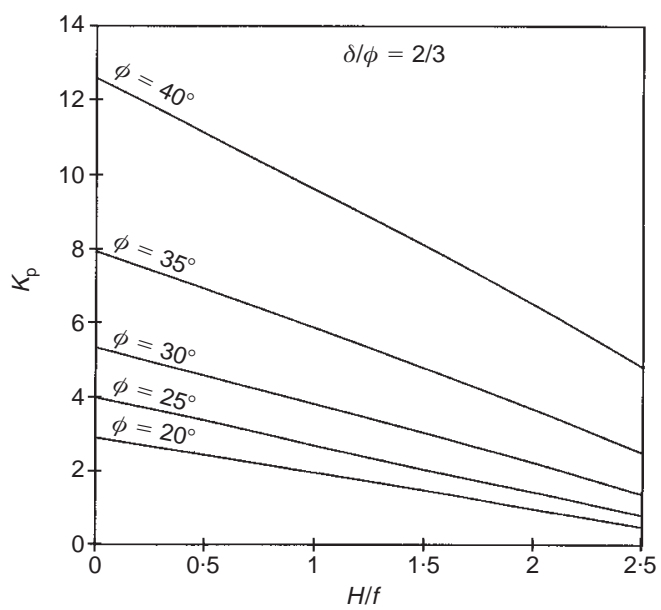

(c) 


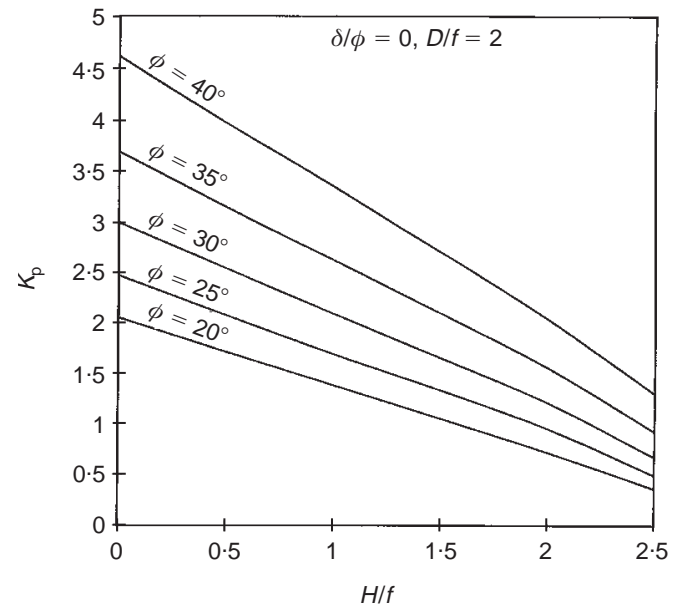

(a)

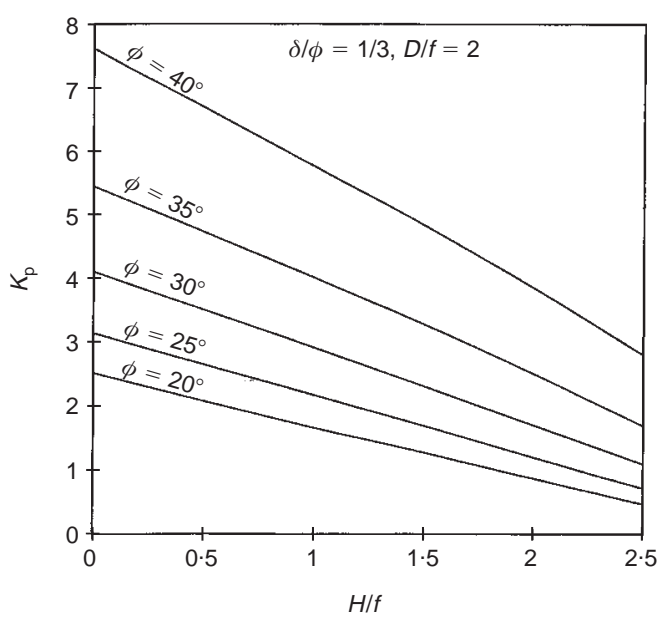

(b)

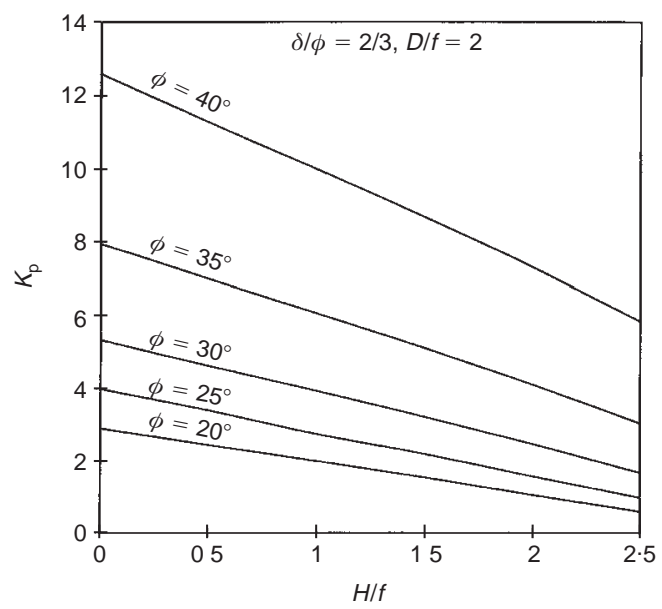

(c)
Case of a single layer of finite depth $D$. The numerical results obtained from the present pro gram have shown that the passive earth pressure coefficient increases with the $D / f$ decrease. The minimum relative depth $D / f$ necessary to obtain the results of the semi infinite case must be greater than or equal to 6 .

Figure 8 shows some charts of the variation of the passive earth pressure coefficient as a function of $H / f$ for different values of $\phi\left(\phi=20^{\circ}, 25^{\circ}\right.$, $\left.30^{\circ}, 35^{\circ}, 40^{\circ}\right)$ and $\delta / \phi(\delta / \phi=0,1 / 3,2 / 3)$ and for $D / f=2$.

Case of a homogeneous and non isotropic soil medium. Figure 9 shows the variation of the passive earth pressure coefficient with $K_{\mathrm{h}} / K_{\mathrm{V}}$ when $\phi=35^{\circ}, H / f=2$ and $\delta / \phi=0,1 / 3,2 / 3$ and 1 . There is a large decrease of the passive earth pressure coefficient up to $K_{\mathrm{h}} / K_{\mathrm{v}}=100$. Beyond this limit, the passive earth pressure coefficient tends to an asymptote. This can be explained by the fact that the equipotential lines in front of the sheet pile become quasi horizontal beyond a certain value of $K_{\mathrm{h}} / K_{\mathrm{v}}$ and, thus, the potential field does not change any more in the zone concerned with the failure mechanism.

Figure 10 shows some charts of the variation of the passive earth pressure coefficient as a function of $H / f$ for different values of $\phi\left(\phi=20^{\circ}, 25^{\circ}\right.$,

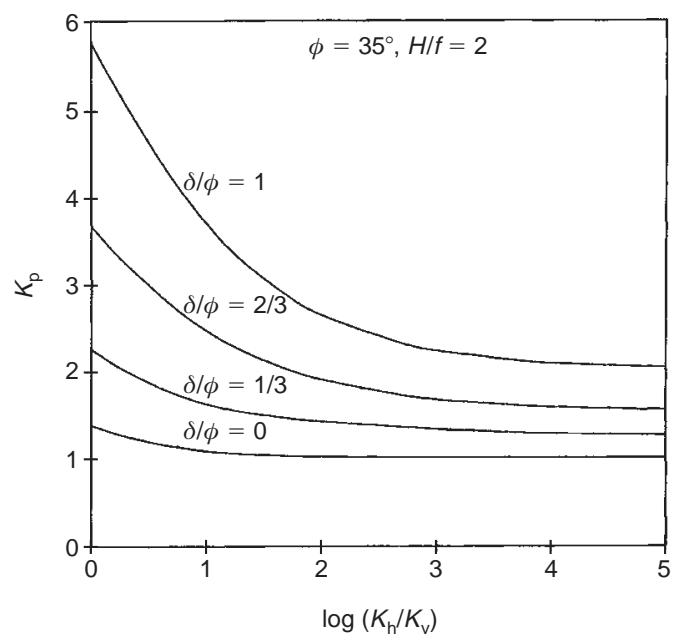

Fig. 9. $K_{\mathrm{p}}$ versus $K_{\mathrm{h}} / K_{\mathrm{v}}$ for $\phi \quad 35^{\circ}$ and $H / f \quad 2$ in the case of a homogeneous and non-isotropic semiinfinite medium

Fig. 8. $K_{\mathrm{p}}$ versus $H / f$ for different values of $\phi$ and $\delta$ in the case of a homogeneous isotropic single layer of finite depth $(D / f \quad 2)$ 


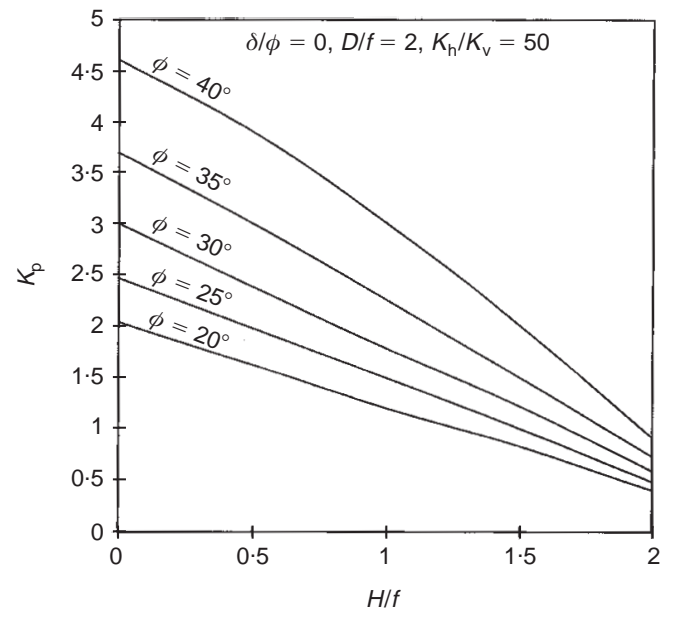

(a)

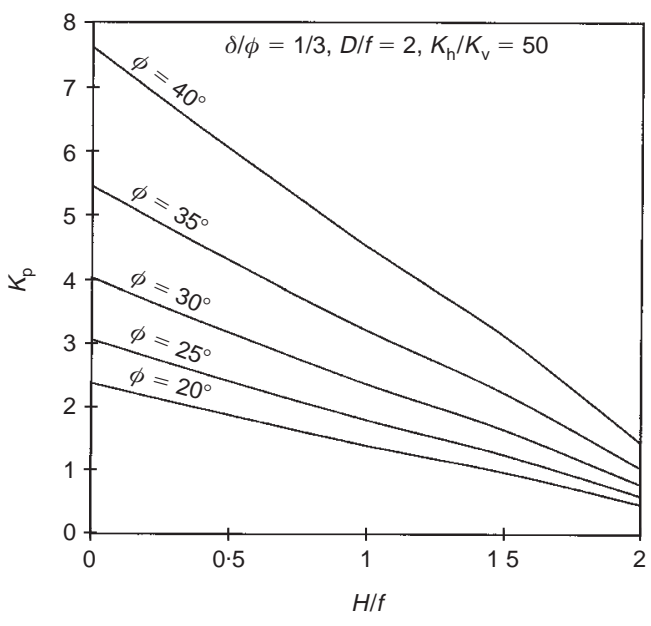

(b)

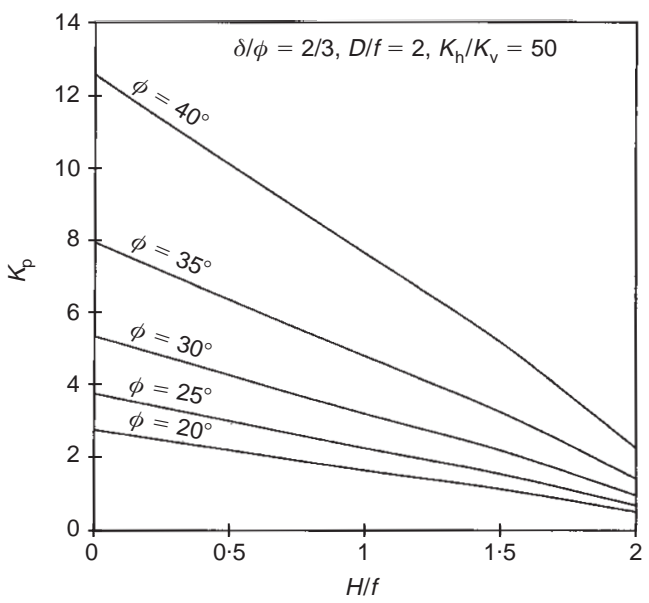

(c) $\left.30^{\circ}, 35^{\circ}, 40^{\circ}\right)$ and $\delta / \phi(\delta / \phi=0,1 / 3,2 / 3)$ and for $D / f=2$ when the permeability ratio $K_{\mathrm{h}} / K_{\mathrm{v}}=50$.

Case of an isotropic two layered soil medium. In this section, we consider the frequent case of a cofferdam driven into a two layered soil medium.

Case where the bottom of the sheet pile lies in the upper layer (case A). Figure 11 shows the case of an isotropic two layered soil medium where the permeability coefficients of the upper and lower layers are respectively $K_{1}$ and $K_{2}$.

Figure 12 shows the variation of the passive earth pressure coefficient with $K_{1} / K_{2}$ when $\phi=35^{\circ}, \delta / \phi=2 / 3$ and $H / f=2$.

For the case of a single layer $\left(K_{1} / K_{2}=1\right)$, the

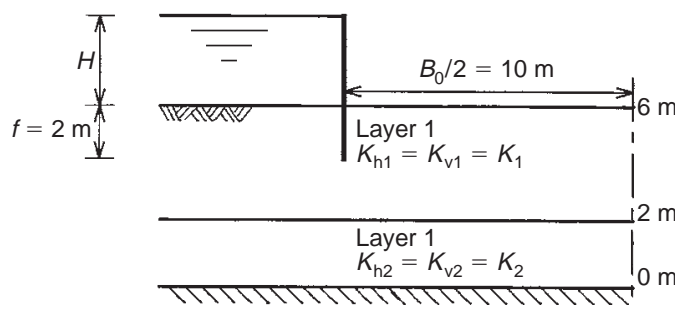

Fig. 11. Case of an isotropic two-layered soil medium: case $\mathbf{A}$

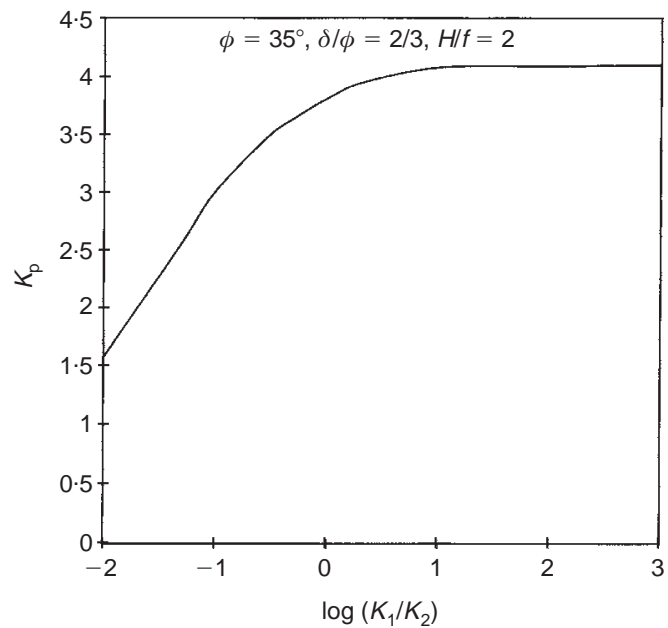

Fig. 12. $K_{\mathrm{p}}$ versus $K_{1} / K_{2}$ for $\phi \quad 35^{\circ}, \delta / \phi \quad 2 / 3$ and $H / f \quad 2$ for case $A$

Fig. 10. $K_{\mathrm{p}}$ versus $H / f$ for different values of $\phi$ and $\delta$ in the case of a homogeneous and non-isotropic single layer $(D / f \quad 2)$ 
passive earth pressure coefficient is equal to $3 \cdot 81$. When $K_{1} / K_{2}>10$, one obtains the passive earth pressure coefficient corresponding to the case of a single layer of limited depth $\left(\phi=35^{\circ}, \delta / \phi=\right.$ $2 / 3, H / f=2$ and $D / f=2)$, since the lower layer can be considered as an impermeable substra tum. Notice, however, that for cases when the lower layer has a greater permeability coefficient than the upper layer $\left(K_{1} / K_{2}<1\right)$, most of the head loss is concentrated in the upper layer, resulting in greater pore water pressures. Consequently, the passive earth pressure coefficient decreases with the $K_{1} / K_{2}$ decrease.

Case where the bottom of the sheet pile lies in the lower layer (case B). Figure 13 shows the case of an isotropic two layered soil medium. The unique difference from the previous case is that the bottom of the sheet pile wall lies in the lower layer.

Figure 14 shows the variation of the passive

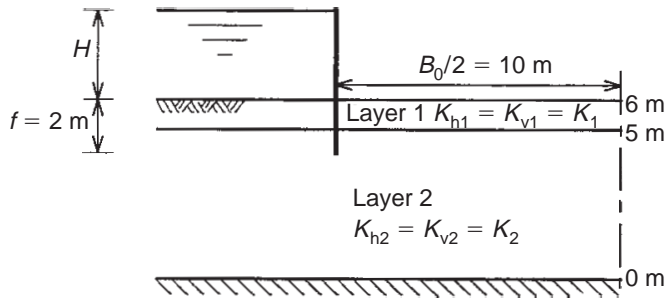

Fig. 13. Case of an isotropic two-layered soil medium: case $B$

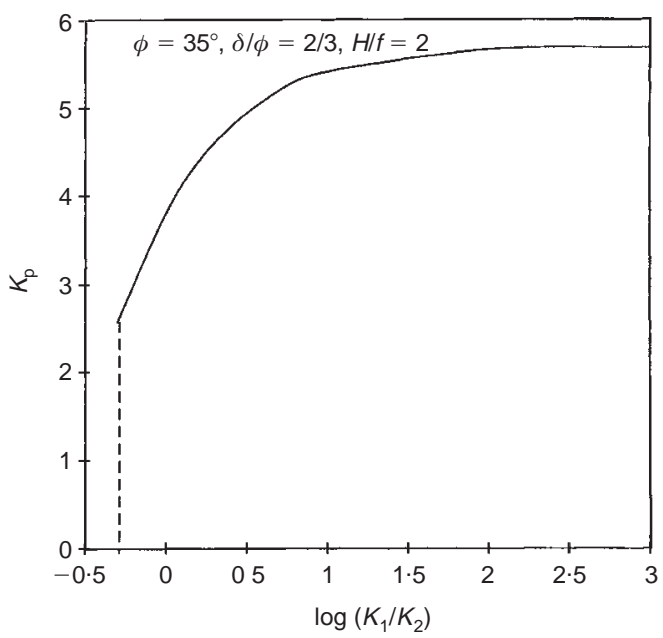

Fig. 14. $K_{\mathrm{p}}$ versus $K_{1} / K_{2}$ for $\phi \quad 35^{\circ}, \delta / \phi \quad 2 / 3$ and $H / f \quad 2$ for case $B$ earth pressure coefficient with $K_{1} / K_{2}$ when $\phi=$ $35^{\circ}, \delta / \phi=2 / 3$ and $H / f=2$.

As in the previous section (case A), the passive earth pressure coefficient in the case of an isotro pic single layer $\left(K_{1} / K_{2}=1\right)$ is equal to $3 \cdot 81$. For $K_{1} / K_{2}>100$, the head loss takes place solely in the lower layer, and the upper layer can be consid ered as a filter increasing the global stability of the soil in front of the sheet pile. Consequently, the increase of the passive earth pressure coefficient with the $K_{1} / K_{2}$ increase is a logical phenomenon. On the other hand, for cases when the lower layer has a greater permeability coefficient than the upper layer $\left(K_{1} / K_{2}<1\right)$, most of the head loss takes place in the upper layer, resulting in a sig nificant reduction of its resultant body force (buoy ant weight + seepage force). Consequently, the passive earth pressure coefficient decreases with the $K_{1} / K_{2}$ decrease. However, it should be noted that this calculation scheme is only valid as long as the vertical hydraulic gradient in the upper layer is smaller than the critical gradient $i_{\mathrm{c}}=\gamma^{\prime} / \gamma_{\mathrm{w}}$, as otherwise failure by heaving of the upper layer will occur due to the fact that the saturated weight of this layer is equal to the resultant of the pore water pressures on the base of this layer. This limitation is shown in Fig. 14 by a vertical dotted line.

\section{CONCLUSION}

The variational limit equilibrium method was applied to the passive earth pressure problem, tak ing into account the seepage flow due to dewater ing. It showed that the failure mechanism, in the general case of non homogeneous and non isotro pic hydraulic properties of the soil medium, is a $\log$ spiral. This method is equivalent to the upper bound method in limit analysis for a rotational log spiral mechanism.

In the case of no seepage flow, the present upper bound solution is the smallest one existing in the literature and is very close to the currently ac cepted results of Caquot \& Kérisel (1948).

In the case of seepage flow, the present mechan ism allowed us to determine the reduction of the passive earth pressures. For the limiting case corre sponding to zero passive pressures, the present mechanism describes the traditional heaving phe nomenon in front of the sheet pile. The numerical results have shown that:

(a) The heaving of a soil mass in front of the sheet pile occurs before the piping phenomenon in the case of a homogeneous and isotropic semi infinite soil medium as long as $\phi$ is smaller than $45^{\circ}$.

(b) The effective passive pressures increase with the decrease of the layer depth in the case of a single layer problem. 
(c) The anisotropy of the permeability coefficient of the soil medium can induce a significant reduction of the effective passive pressures.

(d) The study of the two layered soil medium has shown that: ( $i$ ) for great values of $K_{1} / K_{2}$, when the bottom of the sheet pile lies in the upper layer, one obtains the case of a single layer of limited depth, and when the bottom of the sheet pile lies in the lower layer, the upper layer can be considered as a filter; and (ii) for small values of $K_{1} / K_{2}$, most of the head loss takes place in the upper layer, thus resulting in a significant reduction of the effective passive pressures.

Finally, one can see that the effect of soil aniso tropy and non homogeneity is significant for the reduction of the passive earth pressures. Thus, the assessment of the reduction of the effective passive pressures taking into account these parameters is of great interest in the practice of geotechnical engi neering.

\section{NOTATION}

$B_{0}$ breadth of the double-walled cofferdam

$D$ layer depth

$\mathrm{d} l$ elementary surface along the slip surface

$f$ penetration depth

$H$ total hydraulic head loss

$h, v$ coordinate system whose origin is at point F

$i_{\mathrm{c}}$ critical hydraulic gradient

$K_{1}, K_{2}$ isotropic permeability coefficients of layers 1 and 2

$K_{\mathrm{h}}, K_{\mathrm{v}}$ horizontal and vertical permeability coefficients

$K_{\mathrm{p}}$ coefficient of passive earth pressure

$K_{x}, K_{y}, K_{z}$ permeability coefficients in the principal directions $x, y$ and $z$

$M_{1}$ moment of the force $U_{1}$

$M_{2}$ moment of the pore water pressures acting on the slip surface

$P_{\mathrm{p}} \quad$ effective passive force

$r_{0}, r_{1}$ initial and final radius of the log-spiral slip surface

$U_{1}$ resultant of pore water pressures at the soil structure interface

$u$ pore water pressure

$W_{\text {sat }}$ saturated weight of the soil mass OAB

$X$ distance between the wall and the line of action of the force $W$

$X_{1}$ distance between the bottom of the wall and the normal component of the effective passive force

$X_{2}$ distance between the bottom of the wall and the line of action of force $U_{1}$

$y(x)$ equation of the slip surface in the $(x, y)$ coordinate system

$\dot{y} \quad \frac{\mathrm{d} y}{\mathrm{~d} x}$

$\delta$ friction angle at the soil structure interface $\phi$ angle of internal friction of the soil

$\varphi$ hydraulic head

$\gamma^{\prime} \quad$ submerged unit weight of the soil

$\gamma_{\text {sat }}$ saturated unit weight of the soil

$\gamma_{\mathrm{w}}$ unit weight of water

$\lambda$ relaxation factor

$\lambda_{1}, \lambda_{2}$ Lagrange's undetermined multipliers

$\theta_{0}, \theta_{1}$ angles defining the log-spiral slip surface

$\sigma(x)$ total normal stress distribution along the slip surface

$\sigma^{\prime}(x)$ effective normal stress distribution along the slip surface

$\dot{\sigma}^{\prime} \quad \frac{\mathrm{d} \sigma^{\prime}}{\mathrm{d} x}$

$\tau(x)$ tangential stress distribution along the slip surface

\section{REFERENCES}

Baker, R. \& Garber, M. (1977). Variational approach to slope stability. Proc. 9th Int. Conf. Soil Mech. Found. Engng, Tokyo 2, 912.

Baker, R. \& Garber, M. (1978). Theoretical analysis of the stability of slopes. Géotechnique 28, No. 4, 395 411.

Caquot, A. \& Kérisel, J. (1948). Tables for the calculation of passive pressure, active pressure and bearing capacity of foundations. Paris: Gauthier-Villard.

Castillo, E. \& Luceno A. (1978). One application of the calculus of variations to bearing capacity of foundations. Second Int. Conf. Appl. Numer. Modeling, Madrid 12, 353363.

Castillo, E. \& Luceno, A. (1982). A critical analysis of some variational methods in slope stability analysis. Int. J. Numer Anal. Methods Geomech. 6, 195 209.

Castillo, E. \& Luceno, A. (1983). Variational methods and upper bound theorem. J. Engng Mech., ASCE 109, No. 5, 11571174

Chen, W. F. \& Rosenfarb, J. L. (1973). Limit analysis solutions of earth pressure problems. Soils Found. 13, No. 4, 4560 .

Dorfman, A. G. (1965). Variational method of investigating the stability of slopes. Vop. Geoteckniki Transport 9.

Garber, M. (1973). Variational method for investigating the stability of slopes. Soil Mech. Found. Engng 10, No. 1, 7779 .

Garber, M. \& Baker R. (1977). Bearing capacity by variational method. J. Geotech. Engng Div., ASCE 103, No. GT11, 12091225.

Garber, M. \& Baker, R. (1979). Extreme value problems of limiting equilibrium. J. Geotech. Div., ASCE 105, No. 4, 271282.

Kastner, R. (1982). Excavations profondes en site urbain: problèmes liés à la mise hors d'eau. Dimensionnement des soutènements butonnés. Thèse ès Sciences, INSA Lyon and University Claude Bernard.

Kopacsy, J. (1957). Three-dimensional stress distribution and slip surface in earth work at rupture. Proc. 4th Int. Conf. Soil Mech. Found. Engng 1, 339342.

Leshchinsky, D. \& Baker, R. (1986). Three dimensional slope stability: end effects. Soils Found. 26, No. 4, 98110. 
Leshchinsky, D. \& Reinschmidt, A. J. (1985). Stability of membrane reinforced slopes. J. Geotech. Engng Div., ASCE 111, No. 11, 12851300.

Leshchinsky, D. \& San, K. C. (1994). Pseudostatic seismic stability of slopes: design charts. J. Geotech. Engng 120, No. 9, 15141532.

Leshchinsky, D., Baker, R. \& Silver, M. L. (1985). Three dimensional analysis of slope stability. Int. J. Numer. Anal. Methods Geomech. 9, 199223.

Ly, B. L. (1979). Determination of active earth pressure by a variational method. Third International Conference on Numerical Methods in Geomechanics, Aachen, 11751178

Lysmer, J. (1970). Limit analysis of plane problems in soil mechanics. J. Soil Mech. Found. Div., ASCE 96 , No. SM4, 13111334.

Revilla, J. \& Castillo, E. (1977). The calculus of variations applied to stability of slopes. Géotechnique 27, No. 1,111 .
Shields, D. H. \& Tolunay, A. Z. (1973). Passive pressure coefficients by method of slices. J. Geotech. Engng Div., ASCE 99, No. SM12, 10431053.

Sokolovski, V. V. (1960). Statics of soil media. London: Butterworth.

Soubra, A. H. (1989). Application de la méthode variationnelle au problème de détermination des pressions passives des terres. Influence des forces d'écoulement. Thèse de Doctorat, INSA Lyon.

Soubra, A. H. \& Kastner, R. (1992). Influence of seepage flow on the passive earth pressures. Proceedings of the international conference on retaining structures (ed. C. R. I. Clayton), pp. 67 76. Cambridge: Thomas Telford.

Terzaghi, K. (1943). Theoretical soil mechanics. New York: Wiley.

Ugai, K. (1985). Three-dimensional stability analysis of vertical cohesive slopes. Soils Found. 25, No. 3, 4148. 